\title{
Zur Vorgeschichte zweier Lockescher Begriffe. Von
}

Clemens Baeumker, Straßburg.

II.

S. 166 Anm. lesen wir bei Ueberweg-Heinze: „Die Ausdrücke: Qualitates primae und secundae waren schon bei den Scholastikern üblich; so sagt Bartholomaeus Arnoldi Usingensis (gest. 1532): qualitates primae sunt a quibus aliae fluunt, et sunt quatuor: caliditas et frigiditas, siccitas et humiditas. - Secundae autem sunt quae ab aliis fluunt. Sie wurden von Robert Bayle (in der 9. Auflage stand noch richtig Boyle) auf die verschiedenartigen Qualitäten Descartes' übertragen und von Locke dann aufgenommen (s. Eucken, Gesch. d. philos. Terminol. S. 196).“

Es ist richtig, daß schon in der Scholastik die Ausdrücke qualitates primae und secundae sich finden: nicht erst bei einem so Späten, wie dem für die Entwickelungsgeschichte Luthers in neuerer Zeit zum Vergleich wohl herangezogenen Bartholomaeus von Usingen, sondern schon weit früher.

Die scholastische Unterscheidung geht zurück auf Aristoteles. Ihre Voraussetzungen liegen in der Psychologie und in der Naturphilosophie des letzteren. Die Aristotelische Psychologie gibt be-

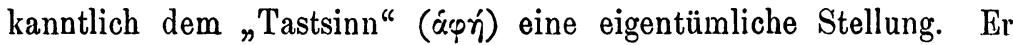
ist der erste Sinn, der Grundsinn. Zunächst subjektiv. Ihn müssen alle empfindenden Wesen ohne Ausnahme besitzen, weil er zur Unterscheidung des als Nahrung Verwendbaren und des das Leben Zerstörenden dient und darum unentbehrlich für die Reaktionen ist, welche das Leben sichern ${ }^{1}$ ). Aber auch objektiv ist er der Grundsinn. Beim Betasten oder Befühlen nämlich erfassen wir unmittelbar, d. h. ohne ein zwischen unserem Leibe und dem wahr-

1) Hauptstelle: De an. III 12, 434 b 10-24. 
genommenen Objekte befindliches äußeres Medium, den wahrgenommenen Körper selbst ${ }^{2}$ ). Deshalb sind die Tastqualitäten Eigenschaften des Körpers als Körper ${ }^{3}$ ).

Eine nähere Analyse dieses Gedankens zeigt uns, wie weit die Unterscheidung primärer und sekundärer Qualitäten schon bei Aristoteles vorgebildet ist ${ }^{4}$ ). Der Tastsinn, so hören wir $^{5}$ ), weist im Gegensatz zu den übrigen Sinnen eine große Anzahl gegensätzlich gepaarter Qualitäten auf. Sieben solcher Paare werden uns aufgezählt: Warm und Kalt, Trocken und Feucht, Schwer und Leicht, Hart und Weich, Schlüpfrig und Spröde, Rauh und Glatt, Dicht und Locker ${ }^{6}$ ), eine Aufzählung, bei der die Frage der Vollständigkeit freilich schon Alexander von Aphrodisias beschäftigte ${ }^{7}$ ). Aber diese verschiedenen Qualitäten sind nicht gleichwertig. Alle jene Gegensätze lassen sich auf zwei zurückführen: Warm und Kalt, Trocken und Feucht, die dann nicht mehr weiter reduzierbar sind ${ }^{8}$ ). Diese sind das Aktive und Passive in den Körpern, aktiv Wärme und Kälte, passiv Trockenheit und Feuchtigkeit ${ }^{9}$ ). Die beiden Gegensätze des Warmen und Kalten, Trockenen und Feuchten werden daher von Aristoteles ausdrücklich als erste Unterschied $\theta^{\mathbf{1 0}}$ ) den übrigen Tastqualitäten gegenüberstellt. „Erste Unterschiede“ nicht

2) De an. III 13, 435 a 14-18.

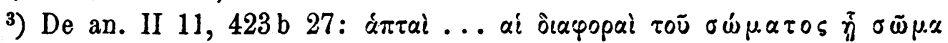
Ebenso De gen. et corr. II 2, 329b 7-11.

$\left.{ }^{4}\right)$ Mit Recht weist hierauf W. Hamilton hin in seiner Abhandlung über die Geschichte des Unterschiedes zwischen primären und sekundären Qualitäten ('The Works of Thomas Reid, by W. Hamilton, 7th ed. Edinburgh 1872 Bd. II S. 816-845), - noch immer dem Besten, was über den Gegenstand geschrieben ist.

5) De an. II 11.

6) De gen. et corrupt. II 2, 329 b 18-20.

7) Alexander bei Philoponus, De gen. et corr. p. $214,23 \mathrm{ff}$. ed. Vitelli. Auch Averroes, De gen. et corr. II com. 15 (ed. Juntina 1562, fol. 374 $A-C)$ beruft sich auf Alexander. Ob Averroes den verlorenen Kommentar Alexanders zu De generatione et corruptione noch in Übersetzung gekannt hat, wie nach Freudenthals Nachweis den gleichfalls verlorenen echten Kommentar zu Metaphysik $\Lambda$ ?

8) De gen. et corr. II 2, 330 a 24-29.

9) Ebd. 329 b 24-32 (Schwierigkeiten bei Pbiloponus p. 216, 23 ff. besprochen).

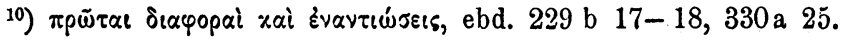


im psychologischen Sinne; die Differenzierung ist vielmehr physikalisch gemeint. Nicht Elementarqualitäten in Empfindungskomplexen sollen jene zwei Gegensatzpaare sein, sondern Grundbestimmungen in der realen Körperwelt. Wenn Aristoteles aus den Gegensätzen der Wärme und Kälte, Trockenheit und Feuchtigkeit die Eigenscliaften der Feinheit und Dichtigkeit, Schlüpfrigkeit und Sprödigkeit, Härte und Weichheit usw. als deren nähere Bestimmungen ableitet ${ }^{11}$ ), so denkt er bei einer solchen Ableitung nicht an die Eindrücke in uns, sondern an das Verhältnis der realen Eigenschaften ${ }^{13}$ ).

Die Tastqualitäten aber sind für Aristoteles die eigentlichen Körperqualitäten. Darum sieht er in den beiden ersten Gegensatzpaaren Warm und Kalt, Feucht und Trocken Grunddifferenzen der körperlichen Naturen und baut auf den vier möglichen Kombinationen der Elemente jener Gegensatzpaare die Unterscheidung der vier körperlichen Elemente auf ${ }^{13}$ ). Die Qualitäten der übrigen

11) Ebd. 329 b 32-330a 24. Schwierigkeiten bei Philoponus p. 223, $9 \mathrm{ff}$. nach Alexander von Aphrodisias

12) Man wende hier nicht ein, daß Wärme und Kälte von Aristoteles an anderer Stelle (Categ. 8, 9a 28-31) unter den $\pi \alpha \vartheta \eta \tau$ geführt werden, die ihren Namen nicht deshalb tragen sollen, als ob hier das Objekt selbst etwas erlitte, sondern weil das Objekt in unseren Sinnen eine Affektion hervorbringe, wie die Süßigkeit des Honigs im Geschmackssinne,

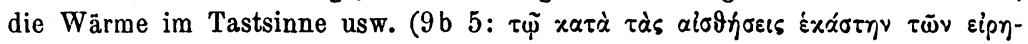

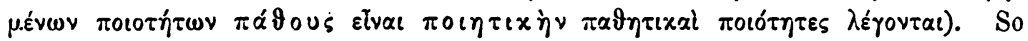

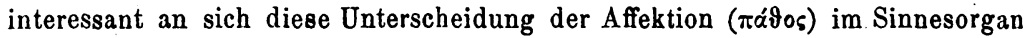

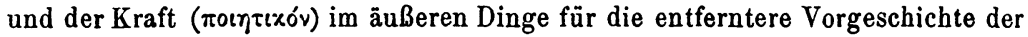
Lehre von der Subjektivität der Sinnesqualitäten sein mag, so kommt sie hier nicht in Betracht, da die Ausfübrungen des Aristoteles gar nicht auf die Frage nach der Realität der Sinnesqualitäten als solcher gehen, sondern nur bestimmen wollen, inwiefern diese von den Dingen aufgenommenen Eigenschaften

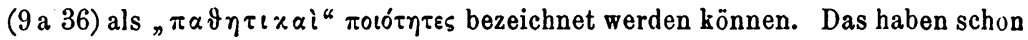
die alten griechischen Erklärer gesehen, weshalb denn Andronikos von Rhodos die Hervorbringung der Affektion im Sinnesorgan als etwas für jene objektiven Qualitäten nur Nebensächliches und nicht Notwendiges betrachten wollte (Simplicius in Categ. 258, 15-21 ed. Kalbfleisch). Andronikos unterschied daher z. B. beim Feuer die objektive Eigenschaft des গвpuóv und das

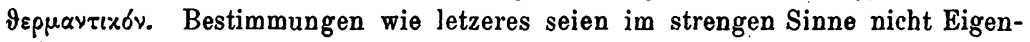

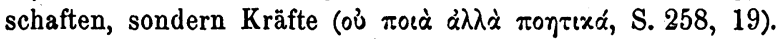

13) De gen. et corr. II, 3. - Die „quinta essentia“ (der unveränderliche Äther) bleibt hier natürlich beiseite. 
Sinne: Farben, Geschmäcke usw., sind nicht Bestimmungen der Körper als Körper. Außer jenen zwei ursprünglichen und ersten

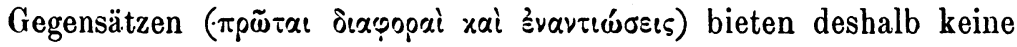
anderen Qualitätengegensätze Elementarbestimmungen der Körper ${ }^{14}$ ).

Wärme und Kälte, Trockenheit und Feuchtigkeit sind somit für Aristoteles erste Gegensätze, und zwar sowohl im Unterschiede von den aus ihnen durch Modifikation entstandenen übrigen Tastqualitäten, wie im Unterschiede von den Qualitäten der übrigen Sinne. Zugleich sind diese ersten Gegensätze das, was den Grundbestandteilen in allen Mischungen und Zusammensetzungen der körperlichen Welt ihre Bestimmtheit verleiht. Freilich widerstrebt es eigentlich den Grundsätzen des Aristoteles, wenn hier Qualitäten in einer Rolle auftreten, die sonst den zur Kategorie der Substanz zu ziehenden Wesensformen anheimfällt. Mittelalterliche arabische und lateinische Erklärer haben sich bemüht, jenen Widerspruch zu entfernen ${ }^{15}$ ); Aristoteles selbst hat ihn anscheinend so wenig beachtet, wie die griechischen Erklärer dies $\operatorname{tun}^{16}$ ).

Der Sache nach hat also bereits Aristoteles erste Qualitäten. Das Wort freilich fehlt noch bei ihm. Er spricht stets von „ersten Gegensätzen“ oder „ersten Unterschieden“. Doch ehe

14) Ebd. II, 2, 329 b 11-13.

15) Averroes sucht De caelo IV com. 40, fol. 267 LM ed. Junt. zu er klären, wie Differenzen von Substanzen aus anderen Kategorien genommen werden könnten, während er Metaph. VIII com. 5, fol. $213 \mathrm{~F}$. leugnet, daß die substanzialen Formen der Elemente in jenen Qualitäten beständen. Thomas von Aquino (De gen. et corr. II lect. 2, a) ist der Meinung, daß Aristoteles zur Unterscheidung der Körperelemente nur deshalb die vier sinnfälligen Qualitäten, welche die unmittelbaren Prinzipien beim substanzialen Wandel seien, anführe, weil die substanzialen Formen als sinnlich nicht wahrnehmbar uns verborgen seien. Ähnlich wie Thomas schon Albert, De gen. et corr. II tract. 2 c. 7 , Bd. IV S. 432 b (ed. Borgnet). Metaph. VIII tr. 1 c. 4, Bd. VI, S. 498 b. Das Gleiche in der Nachscholastik, z. B. Complutenses, De gen. et corr. II, c. 5 q. 1 a. 2.

${ }^{16)}$ Für Aristoteles vgl. mein „Problem der Naterie in der griechischen Philosophie" (Münster 1890) S. 260. Was die griechischen Ausleger anlangt, so nennt z. B. der unter Alexanders Namen gehende Kommentar zu Metaphysik XII das Warme eioos xal ouøia des Feuers (c. 4, S. 680, 24 Hayduck). Philoponus De gen. et corr. II 2, S. 215, 23 Hayduck faßt die vier Tast-

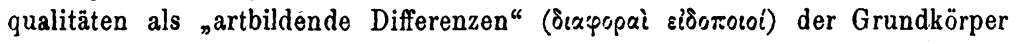


wir das Auftreten auch jenes Wortes nachweisen, sei noch einer anderen Bemerkung des Aristoteles gedacht, die zwar nicht für die Terminologie, wohl aber für die Entwickelung des Begriffes von Bedeutung ist. Ich muß dabei weiter ausholen.

Bekanntlich unterscheidet Aristoteles zwischen den besonderen

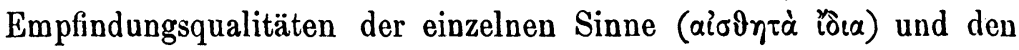

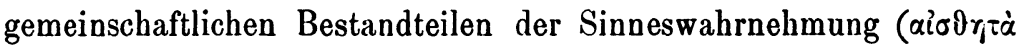
xocvá): eine Unterscheidung, die ja auch Locke - wie vor ihm Herbert von Cherbury ${ }^{17}$ ) - aus der Scholastik herübergenommen hat (Essay II 5), und auf die manche neuere Psychologen, z. B. Ebbinghaus, im Grunde zurückkommen. Solche gemeinschaftliche Wahrnehmungselemente sind bei Aristoteles Bewegung und Ruhe, Zahl, Gestalt, Ausdehnung. Bei Locke fällt diese aus psychologischen Prinzipien gewonnene Gruppe von Wahrnehmungsinhalten zugleich sachlich zusammen mit einer aus erkenntnistheoretischen Gesichtspunkten abgegrenzten Klasse von Eigenschaften, eben mit der der primären Qualitäten, zu der freilich noch eine Tastqualität, die der Undurchdringlichkeit oder Solidität, hinzutritt.

Erkenntnistheoretisch ist auch die Lockes Unterscheidung vorbildende Lehre Demokrits von der Subjektivität der Sinnesqualitäten und die Beschränkung der objektiven Eigenschaften auf die Unterschiede der Gestalt, Anordnung und Lage in der antiken Atomistik.

Die atomistische Lehre nun und seine eigene T'erminologie faßt Aristoteles in einer Art zusammen, die für Locke vorbildlich ist. Die Atomiker, so berichtet er De sensu 4, $442 \mathrm{~b} 10 \mathrm{ff}$., haben die besonderen Sinnesqualitäten (die aiø $\vartheta \eta \tau \dot{\alpha}$ ไồ $\alpha$ ) auf die gemein-

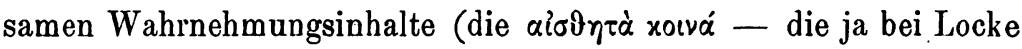
mit den primären Qualitäten sachlich in der Hauptsache zusammenfallen! -) zurückgeführt, z. B. das Weiße und Schwarze auf das Rauhe und Glatte, die Geschmäcke auf die verschiedenen Gestaltungen in den Körperatomen.

Kehren wir zur Entwickelung der Terminologie zurück.

Während Aristoteles nur von „ersten Gegensätzen“ in den Qualitäten spricht, bedienen sich seine mittelalterlichen arabischen

17) C. Güttler, Eduard Lord Herbert von Cherbury. München 1897. S. $53 \mathrm{f}$. 
und lateinischen Erklärer ohne weiteres des Ausdrucks „erste Qualitäten".

So kündigt Averroes seine Ausführungen in De gen. et corr. II (com. 8, fol. 372 E) an, daß er sprechen wolle vom Gegensatz „omnium qualitatum primarum secundum tactum ${ }^{*}$.

Albertus Magnus wiederholt die Aristotelische Lehre und erweitert sie. De gen. et corr. II tr. 1 c. 7 , Bd. IV, S. 423 b Borgnet schließt er in einer sonst wörtlichen Wiedergabe des Aristotelischen Textes (330a 24-29 Bekker) diesen mit leichter Umbiegung: „qua propter necessarium est istas quatuor qualitates (Aristoteles

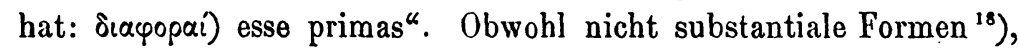
sind jene qualitates primae doch Prinzipien der sinnlich wahrnehmbaren Körper (ebd. c. 5, S. 420a). Nicht bloß die übrigen Tastqualitäten, sondern auch die Qualitäten der anderen Sinne, wie Geschmäcke, Gerüche, Farben (nur mit starker Reserve auch die Töne), werden ausdrücklich auf die ersten Qualitäten als deren Wirkungen zurückgeführt. Wenn dabei die abgeleiteten Qualitäten von Albert auch noch nicht als „sekundäre Qualitäten“ bezeichnet werden, so unterscheidet er beide Klassen doch bereits als „prima sensibilia" und "secunda sensibilia" ${ }^{19}$ ).

Wie Albert, so setat auch Thomas von Aquino in seinem Kommentare zur Aristotelischen Schrift über das Entstehen und

18) Siehe oben Anm. 15. - Durch diese Abweichung wird freilich der reale Wert der ersten Qualitäten etwas herabgesetzt.

19) Albertus Phys. VII tr. 1 c. 5, Bd. III S. 495 b Borgnet: Est enim alteratio proprie secundum prima sensibilia... quae sunt calidum et frigidum et humidum et siccum . . Etiam in secundis sensibilibus est alteratio, sicut in duro et molli, aspero et leni et aliis, quae sunt omnia obiecta tactus. Similiter est in gustativis, eo quod sapores sequuntur qualitates complexionantes (dazu vgl. Phys. Il tr. 2 c. 1 , S. 119 a: est enim complexio qualitas una proveniens ex reciproca actione et passione qualitatum contrariarum in corporibus commixtis), et ideo causantur a primis qualitatibus. Ebenso bei Gerüchen und Farben. Sed in obiectis auditus secus est, quia illa non generantur a qualitatibus primis, sed potius a percutiente et sonante. Doch findet selbst hier eine gewisse Beziehung zu den primären Qualitäten statt; denn: aer est divisibilis per humidum spirituale, quod est in ipso, et ideo quoad hoc aliquam respicit de qualitatibus primis, quia calidum cum humido spirituali puro facit optime sonare. 
Vergehen die Bezeichnung "qualitates primae" als selbstverständlich ein ${ }^{20}$ ).

Ebenso kennt sie Roger Bacon ${ }^{21}$ ). Und Bonaventura ${ }^{22}$ ) stellt unter den „sensibilia propria“ die vier „primariae qualitates" (man beachte das „primariae" statt des sonst üblichen „primae“), welche der Tastsinn wahrnimmt, den übrigen Sinnesqualitäten: Licht, Ton, Geruch, Geschmack, gegenüber.

So ist der Ausdruck „qualitates primae ${ }^{\text {u }}$ oder „primariae schon dem XIII. Jahrhundert ganz geläufig. Die entsprechende Bezeichnung der abgeleiteten Tastqualitäten und der übrigen Sinnesqualitäten als "qualitates secundae" finde ich bei den Autoren jener Zeit noch nicht (obgleich Albert schon von sensibilia prima" und "secunda" sprach), wohl aber im folgenden Jahrhundert.

Heinrich von Hessen ( $†$ 1397) setzt in einer meines Wissens noch nicht gedruckten Schrift „De reductione effectuum in causas communes" (ich benutzte sie in einer Handschrift der Pariser Nationalbibliothek, lat. 14887) auseinander man entschuldige die Ausführlichkeit des frei übersetzenden Referates, da die intrikate und spinöse Sache mit wenigen Worten nicht zu erklären ist - daß, wie die substanzialen Formen, so auch manche akzidentale Formen zu ihrer Hervorführung aus der Potenz der Materie und zu ihrer Erhaltung einer gewissen vorgängigen Disposition der Materie bedürften: eine Disposition, die in der richtigen Proportion zu anderen Qualitäten (nämlich eben zu jenen aus der Materie zu eduzierenden Qualitäten) stehen müsse. Und zwar entständen einige dieser akzidentalen Formen durch die vereinte Einwirkung mehrerer so oder so kombinierter aktiver Qualitäten auf ein so oder so disponiertes Leidendes, andere durch die Einwirkung bloß einer einzigen aktiven Qualität auf ein völlig

20) Tho mas, De gen. et corr. II, lect. 2, d: Ostendit quod non omnes qualitates tangibiles sunt formae perfectivae elementorum, sed solum primae. - So noch mehrmal im gleichen Abschnitt.

21) Roger Bacon, Opus maius V (Perspectiva), dist. 1 c. 3, Bd. II S. 6 ed. Bridges: Aristoteles vult secundo De generatione, quod humidum et siccum uno modo sunt qualitates primae, quae naturaliter elementis debentur.

${ }^{22}$ ) Bonaventura, Itinerarium mentis in Deum c. 2 n. 3. 
disponiertes Leidendes. In dieser Weise entstehende akzidentale Formen seien die Farben, Gerüche, Geschmäcke. Zu diesen zweiton Qualitäten (qualitates secundae) nämlich verhielten sich die ersten (es sind die vier Grundqualitäten Warm, Kalt, Trocken, Feucht) in gewisser Weise wie einfache Elemente, durch deren proportionales Zusammentreten jene zweiten Qualitäten aus der Potenz ihres Subjektes eduziert würden, und zwar entsprechend der jedesmaligen Besonderheit der Proportionen und Kombinationen (der vier Elementarqualitäten). Manchmal werde freilich die Weiße schon durch die Einwirkung der Kälte allein auf ein dazu disponiertes Leidendes hervorgebracht (Heinrich mag an den Schnee gedacht haben), manchmal auch durch die Einwirkung der ersten Wärme ${ }^{23}$ ) auf ein anders disponiertes Leidendes (es mochte die Weißglut des Eisens vorschweben). So würden durch das aktive Zusammenwirken bald von zwei (wie im letzteren Falle, wo ein einziges Tätiges und ein Leidendes zusammenkommen), bald von drei, bald von vier ersten Qualitäten (qualitates primae) die verschiedenen Arten der zweiten Qualitäten (qualitates secundae) hervorgebracht, entsprechend der Verschiedenheit der natürlichen Disposition ${ }^{24}$ ).

23) Die „erste Wärme“ bezeichnet die Wärme als Qualität des Feuerelementes, im Gegensatz zu der Erscheinung der Wärme in dem durch das Feuer Erwärmten. Ähnliches für das humidum und siccum bei Roger Bacon, 0p. maius $\mathrm{V} 1,3, \mathrm{~S} .6$ Bridges.

24) Fol. $76^{\mathrm{v}}$ : Ad declarationem praedictorum est advertendum, quod quaedam formae accidentales ad sui eductionem de potentia materiae exigunt dispositionem praeviam materiae earum, proportionatam ad alias qualitates; quam dispositionem requirunt ad earum conservationem ("earum " bezieht sich nach mittelalterlichem Gebrauch auf quaedam formae accidentales) etiam in subiecto, sicut formae substantiales. Et quaedum fiunt ex concursu plurium activarum qualitatum sic vel sic combinatarum in passum (Leidendes) sic vel sic dispositum; et quaedam ex actione unius qualitatis in passum totaliter dispositum. Et qualitates huius modi sunt colores, odores, sapores; quarum qualitatum secundarum quatuor primae sunt quodam modo ut elementa simplicia, ex quarum proportione (proportionali?) concursu secundum proportionum et combinationum varietatem educuntur huius modi secundae de potentia subiectorum. Quandoque tamen albedo producitur ex actione solius frigiditatis in passum ad hoc dispositum, et quandoque ex actione primi caloris in passum aliter dispositum, ut experimur. Et ita ex concursu activo duarum quandoque primarum qualitatum et quandoque trium et quandoque quatuor 
Hier findet die Lehre von den primären und sekundären Qualitäten in der Form, in welcher die Scholastik sie ausgebildet hat, ihren Abschluß. Die vier Elementarqualitäten stehen nicht nur den übrigen Tastqualitäten, sondern ebenso den Qualitäten der anderen Sinne als primäre gegenüber. Die sekundären Qualitäten werden durch die eigenartigen Kombinationen der primären bewirkt, aber nicht erst - wie in der modernen Lehre - in unseren Sinuen, sondern schon in den Dingen selbst ${ }^{25}$ ).

In dieser Gestalt ist die Lehre dann in der späteren Scholastik verbreitet, wofür der von Eucken angezogene Bartholo maeus Arnoldi von Usingen als Beispiel stehen mag. Noch in der Zeit Lockes findet man sie in den scholastischen Lehrbüchern vertreten. Es sei beispielsweise auf den Dominikaner Goudin ( $†$ 1695) hingewiesen, dessen „Philosophia juxta inconcussa tutissimaque D. Thomae Dogmata quatuor tomis comprehensa" in den Jahren 1671 bis 1692 zehnmal aufgelegt wurde und noch im XIX. Jahrhundert mehrere Neuauflagen erlebte (Paris 1851, Orvieto 1859-60) ${ }^{26}$ ), ja

educuntur variae species qualitatum secundarum secundum varietatem dispositionis naturalis. - Ob Heinrich von Hessen der Erste war, der in dieser Weise die Ausdrücke qualitates primae und qualitates secundae gebraucht, vermag ich natürlich nicht $2 u$ sagen. Genug, daß sie sich bei ibm schon finden.

25) Auf die mannigfache Einschränkungen bedingende Sonderstellung der Töne (sie ist schon durch Aristoteles De anima II 8 angeregt) kann ich bier nicht eingeben (vgl. Anm. 19).

26) Physica III disp. unic. q. 3 a. 2, ed. Orvieto 1859 Bd. III S. 266: Qualitates elementorum aliae dicuntur primae, aliae secundariae. Primae sunt, quae ex aliis non oriuntur, sed immediate formam elementi consequuntur. Secundariae vero, quae ex iis dimanant. Quatuor sunt qualitates primae vulgo notae: calor, frigus, humiditas, siccitas. Die qualitates secundariae sind ( $\$ 4$, S. $276 \mathrm{ff}$.) teils sulche der Elemente, wie Dichtheit und Feinheit, Klebrigkeit und Sprödigkeit, teils solche der Mischungen, wie Gerüche, Geschmäcke, Farben, in gewisser Weise auch die Töne. Diese sekundären Qualitäten der Mischungen entstehen aus den Verbindungen der primären uud sekundären Qualitäten der Elemente und sind daher an Zahl überaus groß. - Wie man sieht, ist es naiv, wenn S. Mosessohn, Robert Boyle als Philosoph (1902), S. 31 meint, Robert Boyle habe jene Termini selbständig gebraucht, da sie als scholastische völlig unbekannt seien. 
noch um die Mitte des verflossenen Jahrhunderts bei den Dominikanern in Rom als Handbuch für die Vorlesungen in Gebrauch war ${ }^{27}$ ).

Wenden wir uns zur neueren Philosophie, so begegnen uns die Ausdrücke "qualitates primae" und „qualitates secundae" sogleich zu Beginn bei Bacon von Verulam, freilich nicht mehr ganz im Sinne der Scholastik, sondern mit leichter Umbiegung hinsichtlich der sekundären Qualitäten. Der Gedanke, daß die Natur bei ihrem Werke gewisse Formen als eigentliches Ziel hervorzubringen strebe, während die übrigen Verschiedenheiten der Dinge nur durch die mannigfachen Hindernisse, welche die Natur bei ihrem Werke finde, und durch das Ineinandergreifen der verschiedenen Formen entständen, habe, meint Francis Bacon, zur Aufstellung primärer Elementarqualitäten geführt, von denen die sekundären Eigenschaften, nämlich die okkulten Qualitäten und die besonderen Wirkungsweisen, wie Anziehung und Abstoßung, Verdünnung und Verdichtung usw. (mit welch letzteren die Medizin sich beschäftigte), unterschieden würden ${ }^{28}$ ). - Wie man sieht, ist auch bei Bacon der scholastische Gedanke in sofern gewahrt, als nicht nur die primären oder Elementarqualitäten, sondern auch die sekundären Qualitäten als real betrachtet und die letzteren zu den ersteren in ein gewisses Abhängigkeitsverhältnis gesetzt werden. Dagegen ist der Inhalt der freilich nur sehr unbestimmt erklärten sekundären Qualitaten ein anderer.

Eine Wendung bringt die Ausbildung der neuen mechanischen Naturansicht. Während in der Polemik die alten Ausdrücke

27) Wie das H. E. Plassmann in seinen „Vorhallen zur Philosophie gemäß der Schule des h. Thomas", Soest 1860, S. X aus eigener Erfahrung berichtet. Ähnlich war in England das 1691 erschienene scholastische Compendium Artis Logicae von Henry Aldrich bis ins XIX. Jabrhundert in Gebrauch und wurde noch 1862 vom Dean Mansel neu herausgegeben.

28) Baco, Novum Organon I 66 (Works, ed. by Spedding, Ellis and Heath, Bd. I S. 176): . . . Atque prima cogitatio qualitates primas elementares, secunda proprietates occultas et virtutes specificas peperit ... At medici, in secundis rerum qualitatibus et operationibus attrahendi repellendi, attenuandi inspissandi, dilatandi astringendi, discutiendi maturandi, et huius modi, operam praestant meliorem. Es ist dann im Folgenden auch von dritten und vierten Qualitäten die Rede. 
lebendig bleiben, werden sie bei der Formulierung der eigenen Ansicht zunächst noch vermieden. So verwendet Pierre Gassend da, wo er polemisiert, die Ausdrücke „qualitates primae“ und „qualitates secundae" ganz in der Weise der Scholastik. In seinem "Syntagma philosophicum" redet er bei der Besprechung des "Materialprinzipes oder der ersten Materie der Dinge “ zuerst (c. 2) von denen, welche als Prinzip eine „materia qualitatibus elementaribus et, ut vocant, primis affecta " aufstellten, wie Thales, Anaximenes usw., dann (c. 3) über die, welche sie auch „aliquibus ex qualitatibus secundis" affiziert sein ließen, wie z. B. Anaxagoras in seiner Homoeomerienlehre. Als primäre Qualitäten werden mit der Scholastik Wärme, Kälte, Feuchtigkeit, Trockenheit, als sekundäre Farbe, Geruch, Geschmack, Licht, Dichtheit, Feinheit und ähnliche aufgezählt ${ }^{29}$ ). Die von ihm gebilligte atomistische Lehre dagegen solle eine qualitätslose Materie (materia ămoıos) aufstellen, da sie den Atomen weder jene sogenannten ersten, noch die zweiten Qualitäten beilege.

Aber nur in der Polemik behält Gassend die scholastische Terminologie bei. Die aristotelisch-scholastische Theorie selbst hat er, wie die Begründer der neuen, mathematisch-mechanisch orientierten Wissenschaft, Galilei, Descartes und Hobbes, völlig verlassen. Nicht nur die $n^{z w e i t e n ", ~ s o n d e r n ~ a u c h ~ d i e ~ „ e r s t e n ~ Q u a l i-~}$ täten“. der Scholastik (wenigstens Wärme und Kälte) sind nach ihm subjektive Einwirkungen der Proprietäten der Atome und Atomverbindungen auf die Sinne. Ganz so faßte bekanntlich Descartes (Princ. philos. IV 198) Licht, Farben, Gerüche, Geschmäcke, Töne, Wärme, Kälte und die übrigen 'Tastqualitäten als subjektive Vorstellungsinhalte auf, denen objektiv nur gewisse Dispositionen der Körper entsprechen; denn nur durch Größe, Figur und Bewegung der kleinsten Partikeln seien die Objekte imstande, unsere Organe zu erregen, nicht aber durch irgendwelche reale „Qualitäten“. Diejenigen realen Eigentümlichkeiten oder Akzidenzien, welche Gassend im Gegensatz zu jenen sogenannten ersten und zweiten Qualitäten der Scholastik als objektive Bestimmungen der Materie

29) Petr. Gassendus, Syntagma philosophicum (in Bd. I der Gesamtausgabe Florenz 1727), Pars II (Physica) sect. I lib. III c. 3, S. 212 b; c. 5, S. 225 a. 
bestehen läßt und die er mit der herkömmlichen Atomistik in untrennbare Akzidenzien (accidentia inseparabilia) und in trennbare oder gemeinsame Akzidenzien (separabilia sive communia) einteilt, je nachdem sie den einzelnen Atomen als solchen (Größe, Figur und Gewicht - woraus die Bewegung - , denen das Solide und darum Undurchdringliche als tragendes Subjekt zugrunde liegt), oder den Atomkomplexen angehören, werden nun $\mathrm{zwar}$ von ihm an manchen Stellen selbst wieder auch "Qualitäten ${ }^{\text {( }}$ genannt ${ }^{30}$ ), indem er den Begriff der Qualitäten überhaupt erweitern will ${ }^{31}$ ); aber als „erste Qualitäten" hat er sie doch nirgendwo bezeichnet; die reale Materie ist nach ihm „qualitätslos" (ämotos, s. 0.). Noch zu fest haftete zu seiner Zeit und in seinen Kreisen an den Ausdrücken „erste und zweite Qualitäten“ die spezifisch scholastische Bedeutung.

Während sonach Gassend nur die Bezeichnung „erste Qualitäten", nicht aber das Wort „Qualität" überhaupt vermeidet, wo er von den „untrennbaren Akzidenzien“ der Korpuskularlehre spricht, drücken die eigentlichen Begründer der mechanischen Naturansicht den Gegensatz gegen die Scholastik auch terminologisch noch bestimmter aus. Bei Descartes (Princ. phil. IV 198, S. 322, 17 4dam) sind die „realen Qualitäten“ Gefährten der nsubstantialen 1 vimen ${ }^{*}$ der Scholastik und werden den mechanischen Bestimmungen der neuen Naturlehre: Größe, Gestalt, Bewegung der kleinsten Teilchen, ausdrücklich gegenübergestellt. Üblich wird für

30) A. a. 0. c. 6 , S. 233 b: Cum autem praeter hanc substantiae, seu identitatem mavis seu similitudinem dicere, attribuantur atomis qualitates quaedam sive accidentia, quarum ... alia sunt inseparabilia, . . . alia separabilia et ... vulgo accidentia cornmunia dicantur. Vgl. Philosophia Epicuri, pars II, sect. I e. 6, Bd. III S. 15 a, wo den Atomen „proprietates et qualitates quaedam" beigelegt werden.

31) Syntagma a. a. 0. lib. VI, c. 1, Bd. I S. 327 b. Auch Größe, Gestalt, Bewegungskraft, die „okkulten “ Qualitäten der Sympathie und Antipathie, des Magnetismus und der Elektrizität, und noch vieles andere sollen unter dem Namen „Qualität" begriffen werden. - Den Begriff der Qualität bei Gassend und sein Verhältnis zum scholastischen Begriff erörtert Paul Pendzig in seiner gerade erscheinenden Schrift: Pierre Gassendis Metaphysik und ihr Verhältnis zur scholastischen Philosophie. Bonn 1908 (Renaissance und Philosophie. Beiträge zur Geschichte der Philosophie, berausgegeben von A. D yroff. I), S. $28 \mathrm{ff}$., $119 \mathrm{ff}$. Auf die uns beschäftigende terminologische Frage ist Pendzig nicht eingegangen. 
die letzteren der (auch Gassend geläufige) Name: erste oder untrennbare Akzidenzien. So hatte auch Galilei im "Saggiatore" (1623) den fälschlich als real betrachteten „Affektionen“ (bei ihm im Sinne von $\pi \dot{\theta} \vartheta \eta$ ) und „Qualitäten" (affezioni e qualità) die allein realen „ersten Akzidenzien " (primi accidenti) gegenüberstellt ${ }^{32}$ ). Als solche untrennbare Akzidenzien bezeichnet auch $\mathrm{Hob}$ bes Ausdehnung (die bei ihm mit der Größe zusammenfällt) und Figur, indem er unter naccidens" die Weise (modus), den Körper vorzustellen, versteht ${ }^{33}$ ).

Die Verbindung der scholastischen Terminologie mit der neuen Naturansicht wird eingeleitet durch Robert Boyle ( $\dagger$ 1691) in seiner 1666 erschienenen Abhandlung über den Ursprung der Qualitäten und Formen ${ }^{34}$ ), auf die als die wahrscheinliche Quelle von Lockes Terminologie, lange vor Eucken, schon W. Hamilton aufmerksam gemacht hat ${ }^{35}$ ).

Boyle steht Gassend nahe. Freilich stimmt seine Theorie keineswegs völlig mit der Gassends überein. Handelt es sich hier doch um Errungenschaften, die von verschiedenen Gesichtspunkten aus Galilei, Gassend, Descartes, Hobbes gemeinschaftlich gewinnen ${ }^{36}$ ) und die bei jedem bedeutenderen Geiste - so auch bei Boyle eine eigenartige Ausprägung erhalten. Und ob Boyle seine Kenntnis der scholastischen Terminologie in diesem Falle Gassend entnommen hat ${ }^{37}$ ) oder direkt einem der späteren Scholastiker, mit denen er wohl

32) Galilei, Opere, Firenze 1842-1856, Bd. IV S. 333, 334.

${ }^{33}$ ) Hobbes, De corpore II p. $8 \S 2,3,4$, ed. Molesworth, Latin, Bd. I S. 92und 93. Vgl. Anm. 43.

34) Rob. Boyle, Considerations and Experiments touching the Origin of Forms and Qualities, 0xford 1666, in der hier benutzten fünfbändigen Gesamtausgabe von R. Boyle's Works, besorgt durch Thomas Birch, London 1744, in Bd. II S. $460 \mathrm{ff}$. (Die dreibändige Ausgabe der „Philosophical Works“ Boyles von Peter Shaw - sie liegt mir in der zweiten Auflage, London 1738, vor - enthält nur einen verkürzten Text). Außerdem habe ich auch die unter dem Titel: „Origo formarum et qualitatum juxta philosophiam corpuscularem considerationibus et experimentis illustrata" $\mathrm{zu}$ Genf 1688 erschienene lateinische Übersetzung herangezogen.

35) A. a. 0. S. 833 a.

36) Noch manche andere kommen in Betracht, wie Derodon, Glanville, de la Forge, über die Hamilton a. a. 0. S. $832 \mathrm{ff}$. handelt.

${ }^{37}$ ) Wie Johann Meier in seinem Aufsatz über „Robert Boyles Naturphilosophie. Mit besonderer Berücksichtigung seiner Abhängigkeit von Gassendi 
bekannt ist ${ }^{38}$ ), wird sich nicht mit Sicherheit entscheiden lassen. Jedenfalls kennt er die „peripatetische“ Lehre von den „ersten Qualitäten", die sich in den vier Elementen Feuer, Wasser, Erde, Luft wie substantiale Formen verhalten sollen ${ }^{39}$ ).

Boyles eigene Theorie der Qualitäten beruht auf der Korpuskularphysik ("the Corpuscularian doctrine of qualities", Works III, 586 a) und will darum eine mechanische Erklärung der Qualitäten geben $\left.{ }^{40}\right)$. Die eine und allgemeine Materie, unter welcher die ausgedehnte, teilbare und undurchdringliche Substanz zu verstehen ist $^{41}$ ), zerfällt nach Boyle in kleinste Teile (minute parts or minima

und seiner Polemik gegen die Scholastik “, Philos. Jahrbuch, hrsg. von Gutberlet, XX (1907) S. 75 f. meint.

38) Vgl. Origin of Forms and Qual. S. 461 b: the doctrine of many modern schoolmen (die lateinische Übersetzung hat p.4: quod aiunt moderniores Scholastici). S. 462 a: the lately mentionned scholastick opinion. S. 476, a werden den griechischen Kommentatoren des Aristoteles his Latin followers, the schoolmen (scholastici die Übersetzuug p. 31) und others gegenübergestellt. Ebendort S. 476 b spricht er von der ,controversy betwixt us and the schools in this, wether or no the forms of natural things ... be in generation educed, as they speak, out of the power of the matter", und fübrt S. 477 a ganz richtig eine Ausflucht an, durch die, the modern schoolmen " sich zu helfen suchen, und Ähnliches an zabllosen anderen Stellen der Schrift. Scotus und Suarez werden S. 529a erwähnt; "the famous Jesuit Suarez ${ }^{*}$ und seine vielgelesenen Disputationes metaphysicae, nebst dessen „learned Protestant annotator Revius" auch S. 465, Anm. An letzterer Stelle auch Ariaga und Hurtado.

${ }^{39}$ ) Ebd. S. 480 a: . . . there is not an inconsiderable party among the Peripateticks themselves who maintain, that in the elements the first qualities (as they call them) are instead of forms, and that the fire (for instance) hath no other form than heat and dryness, and the water than coldness and moisture. - So lehrten in der Tat die griechischen Peripatetiker, während in der lateinischen Scholastik die substanziale Form von den primae qualitates unterschieden wurde. S. oben Anm. 15 und 16.

$\left.{ }^{40}\right)$ Experiments, Notes, etc. about the Mechanical Origin or Production of Divers Particular Qualities, Works, Bd. III S. $566 \mathrm{~b}$ : In my explications of qualities, I pretend only, that they may be explicated by mechanical principles.

$\left.{ }^{41}\right)$ Origin etc. S. $460 \mathrm{~b}$ : there is one catholick or universal matter common to all bodies, by which I mean a substance extended, divisible and impenetrable. - Die Undurchdringlichkeit ist also nicht erst von Locke den ursprünglichen Eigentümlichkeiten hinzugefügt worden, wie G. Geil, Über die Abhängigkeit Lockes von Descartes, Straßburg 1887, S. 85 f.,

Archiv fur Geschichle der Philosophie. XXI. 4. 
naturalia II, 475a), in Korpuskeln oder Atome, deren jedem drei notwendige Attribute zukommen: Größe, d. h. bestimmte Größe (magnitude, or rather size, d. h. a determined quantity, S. $461 \mathrm{~b}$; auch bulk, S. 465 b), Figur (figure or shape) und Bewegung oder Ruhe (motion or rest). Von diesen drei Eigentümlichkeiten werden diebeiden ersten, wie bei Gassend und Hobbes, „untrennbare Akzidenzien “ ${ }^{42}$ ) (inseparable accidents) genannt ${ }^{43}$ ), wohingegen Bewegung und Ruhe wechseln können, wenn auch einer dieser Zustände immer vorhanden sein muß. Während Größe und Figur schon den einzelnen Korpuskeln eignen, sind Lage (posture, S. 466 a) und Ordnung (order) reale Eigentümlichkeiten der Verbindungen von Korpuskeln. wozu dann noch die besondere Disposition der Teile, die „Textur ${ }^{\mathfrak{c}}$ (texture) hinzutritt. Alle diese Eigenschaften sind „mechanische (wir würden sagen: quantitative) Attribute ${ }^{44}$ ). Auch die Organe des menschlichen Leibes haben ihre verschiedene "Textur" und werden dadurch befähigt, durch die Figur, Gestalt, Bewegung un! Textur der äußeren Körper erregt zu werden und dadurch „Eindrücke" (impressions) von ihnen aufzunehmen. So entstehen die Sinnesqualitäten (sensible qualities): Licht, Wärme, Ton, Gerucı usw. ${ }^{45}$ ) Sie sind als solche nicht reale, physische Wirklichkeitea in der Materie und können daher den Körpern selbst, welche durć

meint. Um von Gassend (s. o.) zu schweigen, betrachtet sie selbst Descarts als in der „wahren “ Ausdehnung des Körpers eingeschlossen: Meditat., Res\}. VItae n. 9, ed. Adam Bd. VII S. 442, 6: vera enim corporum extensio tals est, ut omnem partium penetrabilitatem excludat.

42) „Akzidenzien“ sollen diese Bestimmungen bleiben, weil die Figur jenor Körperchen zwar nicht von Naturagenzien, wohl aber im I)enken verändet und das Körperchen selbst im Denken noch weiter geteilt werden könne, ohre daß die ganze Wesenheit der Materie zerstört werde. - Man beachte, da nicht die Ausdehnung (extension), sondern nur die bestimmte Größe und Figur als Akzidens bezeichnet wird. Nach dieser Seite hin liegt also ein Gegensaz zu Descartes nicht vor.

43) Ähnlich Hobbes, De corpore II p. $8 \S 3$ ed. Molesworth Bd. I S. $92 \mathrm{f}$ : ... quaedam accidentia abesse a corpore sine interitu eius non possun; nam corpus sine extensione (die $\S 4$ der magnitudo gleichgesetzt wird) at sine figura omnino concipi non potest. Doch ist hier der Unterschied, do Boyle zwischen extension und size aufstellt, noch nicht gemacht.

44) A. a. O. S. 463 a (s. Anm. 47).

45) A. a. O. S. $466 a$. 
ihre primären Affektionen diese Qualitäten in den Sinnen hervorbringen ${ }^{46}$ ), nur so uneigentlich beigelegt werden, wie wir diesen auch negative Eigenschaften zusprechen. Hier, in den Körpern selbst, korrespondieren ihnen nur gewisse mechanische Modifikationen der Materie: bestimmte Modifikationen der Größe, Figur, Textur u. dgl. ${ }^{47}$ )

In dieser Weise führt Boyle den Unterschied zweier Gruppen von Eigenschaften, von realen und von bloß subjektiven, den die neue Naturphilosophie allgemein aufstellte, durch. Auf denselben wendet er nun die scholastische Terminologie an. Freilich nur zum Teil. Für die Sinnesqualitäten, die als solche nicht real sind, sondern erst durch die besonderen Modifikationen der ursprünglichen Eigentümlichkeiten der Materie in den Sinnesorganen hervorgebracht werden, will er die Bezeichnung "sekundäre Qualitäten“ verwenden - er führt den Ausdruck bezeichnender Weise mit einem: „if I may so call them" ein $-{ }^{48}$ ); aber jene realen Eigentümlichkeiten: Größe, Figur, Bewegung oder Ruhe, Lage, Ordnung, Textur ${ }^{9}$ ), nun entsprechend auch „erste Qualitäten“ zu nennen,

${ }^{46}$ S. 461 a: Wether these accidents may not conveniently enough be called the moods or primary affections of bodies, to distinguish them from those less simple qualities (as colours, tastes and odours), that belong to bodies upon their account ... Von der besonderen Textur des Ganzen und den „mechanical affections“ seiner kleinsten Teile hängt nämlich die Wirkungsweise der Körper ab: S. 481 a. Wir müssen deshalb suchen, für alle besonderen Qualitäten mechanische Ableitungen (mechanical accounts) $z u$ geben: Works III, 569 a. Vgl. 566 b (Anm. 40). Vgl. ferner Anm. 56.

47) A. a. O. S.463a: And proportionably hereto, I do not see, why we may not conceive, that as to those qualities (for instance) which we call sensible, though, by virtue of a certain congruity or incongruity in point of figure or texture (or other mechanical attributes) to our sensories, the portions of matter they modify are enabled to produce various effects, upon whose account we make bodies to be endowed with qualities; yet they are not in the bodies, that are endowed with them, any real or distinct entities, or differing from the matter itself, furnished with such a determinate bigness, shape or other mechanical modifications.

${ }^{48)}$ A. a. O. S. 466 a (siehe unten Anm. 57). Auch die lateinische Übersetzung von 1688 hat dies "si ita dicam" (S. 13). Bei Shaw (Bd. I, S. 203) dagegen sind jene Worte ausgelassen: bezeichnend für die mittlerweile erfolgte Einbürgerung der neuen Terminologie.

49) So werden sie S. 468 b zusammenfassend aufgezählt. 
trägt er, wie Gassend und Galilei, offenbar Bedenken. Auch er, wie Gassend, fühlt aus dem Worte „Qualität" noch Nebenbeziehungen heraus. Wenn er auch keine eigentliche Definition der Qualität geben will - die in der Scholastik herkömmliche des Aristoteles ${ }^{50}$ ) verwirft er als Tautologie - , so steht ihm doch fest, $\mathrm{da} B$ alle Qualitäten direkt oder indirekt (letzteres sind die „tertiären Qualitäten " Lockes) Inhalte der Sinneswahrnehmung sein müssen ${ }^{51}$ ). Hinsichtlich jener primären Eigentümlichkeiten, die ja in erster Linie als Bestimmungen der für sich allein nicht wahrnehmbaren kleinsten Körperchen in Betracht kommen, trifft dies nicht zu.

Ausdrücklich verwirft deshalb Boyle in einer späteren (1671 erschienenen) Schrift die Bezeichnung von Größe, Figur, Bewegung oder Ruhe als „Qualitäten“ ${ }^{52}$ ). Er will sie „Modi“ (moods oder

50) Doch hat schon die Scholastik das Bedürfnis einer Erklärung empfunden, die nicht bloB, wie die Aristotelische (Categ. 8, p. 8 b 2j:

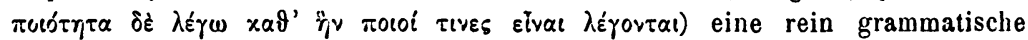
ist. Vgl. z. B. Suarez, Metaph. disp. XLII sect. 1. Auch Boyle ist nachträglich darauf aufmerksam geworden (S. 465 Anm.). (Man beachte übrigens,

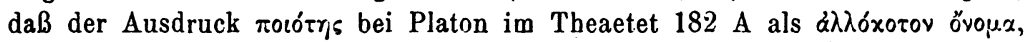
als „fremdartiges Wort", auftritt, woraus schon ein Scholiast schloß, daß Platon den Terminus zuerst eingeführt habe. Noch Aristoteles konnte deshalb sehr wohl das Bedürfnis einer grammatischen Erklärung verspüren und in diesem Sinne die vielgetadelte Definition in den Kategorien aufstellen.)

51) S. 465 a (qualities) being immediately or reductively the objects of sense ... Das „reductively" erklärt S. $466 \mathrm{~b}$ : Nor do I say, that all qualities of bodies are directly sensible; but I observe, that when one body works upon another (z. B. die glühende Kohle, welche nicht nur in der Hand Wärme hervorbringt, sondern auch Wachs und Eis schmilat), the knowledge we have of their operation proceeds either from some sensible quality, or some more catholick affection of matter (man beachte, daß hier "affection", nicht "quality" steht), as motion, rest, or texture, generated or destroyed in one of them. - Erklärung und Beispiele zeigen deutlich, daß es sich bei den indirekt wahrgenommenen „Qualitäten " nicht um die primären, sondern um die tertiären Qualitäten Lockes handelt. Wo Boyle selbst die „Figur" als Qualität bezeichnet (z. B. S. $461 \mathrm{a}-\mathrm{b}$ : shape and other qualities) meint er nicht die Figuren der Atome, sondern die Gestalt zusammengesetzter Körper, die Figur im Sinne der vierten Art der Qualität bei Aristoteles (Categ. 9, p. 10 a $11 \mathrm{ff}$.) und den Scholastikern (an der angeführten Stelle die Form eines Messers oder Rasiermessers, die ein solches zum Schneiden befähigt).

52) Boyle, History of Particular Qualities, Oxford 1671 (lateinisch Genf 1677) ch. 1 (Works III, 73 a): And there are some otherr attributes, namely, 
modes) oder „erste Affektionen “(primary affections) der Materie genannt wissen ${ }^{53}$ ). Auch ${ }_{n}$ Attribute ${ }^{54}$ ) (attributes) heißen sie oder auch — wie bei Galilei ${ }^{55}$ ) — „erste Akzidenzien“ (primary accidents $\left.{ }^{56}\right)$. So auch an der bekannten Stelle, von welcher der eine dem anderen nachschreibt, daß dort die scholastischen Ausdrücke „primäre und sekundäre Qualitäten“ auf die verschiedenartigen Qualitäten des Descartes übertragen würden. In Wahrheit werden dort die „einfacheren und mehr ursprünglichen Affektionen" (simpler and more primitive affections) und die $n$ sekundären Qualitäten " (secondary qualities) gegenübergestellt ${ }^{57}$ ).

size, shape, motion, and rest, that are wont to be reckoned among qualities which may more conveniently be esteemed the primary modes of the parts of matter; since from these simple attributes, or primordial affections all the qualities are derived.

53) Origin, S. 461 a: Moods or primary affections of bodies (s. Anm. 46). On the Systematical or Cosmical Qualities of Things, Works III, 82 a: „primitive modes and catholick affections of matter itself". „Modes" steht auch III, 73 a (Anm. j2).

54) „Mechanical attributes“ II, $463 \mathrm{a}$; „simple attributes" III, 73 a u. o.

55) Irrig ist, was A. Riehl, Der philosophische Kritizismus ${ }^{2}$, Bd. I S. 43 über den Unterschied von Galilei und Boyle bemerkt. Boyle spricht zwar auch von „ersten Qualitāten“, aber nur, um die Lehre der Aristoteliker zu bezeichnen; s. oben Anm. 39. Im übrigen hebt Riehl vortrefflich den Grundfehler in der üblichen Auffassung von Lockes Qualitātenlehre hervor. Schon Hartenstein erhob 1861 Klage über die mannigfachen Mißdeutungen der Lockeschen Lehre.

56) Boyle, Origin etc., S. 466 b Whereas indeed ... there is in the body, to wich these sensible qualities are attributed, notbing of real and physical, but the size, shape, and motion, or rest, of its component particles, together with that texture of the whole, which results from their being so contrived as they are; nor is it necessary they should have in them any thing more, like to the ideas they occasion in us, those ideas being either the effects of our prejudices or inconsiderateness, or else to be fetched from the relation, that happens to be betwixt those primary accidents of the sensible object, and the peculiar texture of the organ it affects.

57) Origo qual. et form. p. 13: Dari nulla alia in corporibus accidentia praeter colores, odores etc. ideo abfuit ut affirmarem, ut e contra haud semel probaverim, simpliciores esse et magis primarias affectiones, de quibus secundariae illae (si ita dicam) qualitates dependent; mutuas autem corporum mutationes inde oriri (Lockes tertiäre Qualitäten!) mox videbimus. Im englischen Original, S. 466 a: I say not, that there are no other accidents in bodies than colours, odours, and the like; for I have already 
Denn die "primären Affektionen" sind Eigentümlichkeiten der Körper, die sekundären Qualitäten dagegen sind nicht Eigenschaften der Dinge selbst, sondern durch die Modifikationen der primären Qualitäten bewirkte „Ideen ${ }^{4}$ in uns $^{58}$ ). Nebenbei zeigt sich hierin, wie die aristotelisch-scholastische Bestimmung des Verhältnisses von ersten und zweiten Qualitäten, nach der die letzteren aus den ersteren in den Dingen selbst physisch hervorgehen, von Boyle, soweit die Qualitäten selbst - nicht deren Grundlage - in Betracht kommen, verlassen ist. Bei Locke werden wir anderes finden.

Nicht schon bei Boyle finden wir also die neue Terminologie formell durchgeführt. So bleibt John Locke der Erste, welcher die Bezeichnungen „primäre und sekundäre Qualitäten“ (original or primary qualities, secondary qualities, Essay II 8, 11-12 u. ö.) im neuen Sinne ausdrücklich verwendet hat. Natürlich schloß er sich dabei an seinen Freund ${ }^{59}$ ) Boyle an, mit dessen Schriften er wohl bekannt ist ${ }^{60}$ ) und den er an mehr als einer Stelle rühmend erwähnt ${ }^{61}$ ). Waren es doch nur Rücksichten auf aristotelisch-schola-

taught, that there are simpler and more primitive (Boyle sagt nicht hier einmal „primary"!) affections of matter, from which these secondary qualities, if I may so call them, do depend: and that the operations of bodies upon one another spring from the same (Lockes tertiäre Qualitäten; vgl. Anm. 51), we shall see by and by.

58) S. Anm. 56.

59) Fox Bourne, The Life of John Locke, 1876, Bd. I, S. 133. Noch an Boyles Totenbett war er: ebd. II 232.

$\left.{ }^{60}\right)$ Locke besprach Schriften von Boyle in der „Bibliothèque universelle“ (Fox Bourne II $44 \mathrm{f}$.), gab auch Aufzeichnungen Boyles nach dessen Tode unter dem Titel „A general History of the Air ${ }^{4}$ heraus (ebd. II 225). Wie auch Kleinigkeiten in Lockes Essay durch Boyle angeregt sind, zeigt vo n Hertling (John Locke und die Schule von Cambridge, Freiburg 1892, S. 263) an der an Boyles "Exercitationes de utilitate philosophiae experimentalis", Genf 1694 (englisch als „Some Considerations touching the Usefulness of Natural Philosophy“ schon 1663), S. 61 (auch S. 9 und 76!), erinnernden Erwähnung der Straßburger NIünsteruhr bei Locke, Essay III ch. $6, \S 3$ und 9 . (In der englischen Gesamtausgabe von Boyles Werken, London 1744, finden sich die Stellen Bd. I S. 424 b, 446 a und 452 a.)

${ }^{61}$ ) Nicht jeder darf hoffen, ein Boyle oder Sydenham zu sein", heißt es in dem dem Essay voraufgeschickten „Briefe an den Leser" (Essay, ed. Fraser, Bd. I. S. 14). In der zweiten Replik Lockes an den Bischof von Worcester wird Boyle als „excellent person and philosopher", „the learned Mr. Boyle" 
stische Begriffsfassungen, welche Boyle abgehalten hatten, den „zwciten Qualitäten“, wie die Konsequenz forderte, nun auch „erste Qualitäten" gegenüberzustellen. Für Locke, dem die alte Metaphysik und Physik schon ganz fern steht, fielen diese Bedenken fort.

Daß aber Locke in der Aufstellung der ersten Qualitäten ganz Boyle folgt, erhellt am besten aus dem Umstande, daß deren ziemlich wirre Aufzählung erst aus Boyle, dem sie am nächsten steht - weit näher, als der Darstellung bei Gassend ${ }^{62}$ ), dem im ïbrigen Locke gleichfalls manches verdankt ${ }^{63}$ ) - rechtes Licht erhält. Undurchdringlichkeit (solidity), Ausdehnung (extension), Größe (size) oder Masse (bulk), Gestalt (figure), Bewegung und Ruhe (motion or rest, mobility), Zahl (number of parts IV 3,15, meist einfach number) und Textur (texture) werden aufgeführt, bald diese, bald jene, ohne feste Konsequenz. Bald werden diese Eigenschaften oder doch bestimmte von ihnen den Körpern im ganzen beigelegt, die groß genug sind, um wahrgenommen zu werden (II 8, 22), bald - und dieses ist das Gewöhnliche - treten sie als Bestimmungen der kleinsten Teilchen auf, aus denen Locke, entsprechend der Korpuskularphilosophie seiner Zeit, die Körper bestehen läßt. Denn wenn Locke auch über naturwissenschaftliche Hypothesen prinzipiell keine Entscheidung geben will (IV 3, 16), so hält er ein Eingehen auf naturwissenschaftliche Lehren doch für nötig, um den Unterschied zwischen objektiven Eigenschaften der Körper und unseren Sinnesvorstellungen begreiflich zu machen (II 8, 22), und hier huldigt er der Korpuskularhypothese (corpuscularian hypothesis IV 3, 16). Alles das wird klar durch Boyles „Korpuskulardoktrin der Qualitäten" (s. o.); welche die von Locke angeführten primären Qualitäten in allgemeine Eigenschaften der Materie, Akzidenzien

eingeführt (Works, $12^{\text {th }}$ ed., 1824, Bd. III, S. 364) und mit Galilei, Bacon und Newton als Entdecker neuer Wahrbeiten gepriesen, die man nicht deshalb zurückweisen dürfe, weil sie nicht schon von den Griechen gelehrt seien (S. 402).

62) Ein Hauptunterscbied zwischen Locke und Gassend liegt darin, daß letzterer das Gewicht (als Ursache der Bewegung) unter den untrennbaren Akzidenzien aufführt, wäbrend es bei Locke völlig übergangen ist. - Auch die Bezeichnungen der primären Qualitäten bei Locke stimmen völlig mit denen bei Boyle, nicht mit denen Gassends, überein.

63) Fox Bourne II, S. 91 . 
der einzelnen Korpuskeln und Eigenschaften der Atomverbindungen gliedert. So findet auch die "Textur" ihren systematischen Platz, die bei Locke (II $8,10,14,18,19$. IV 3, 11), wie bei Boyle, als Grand der sekundären Qualitäten des öfteren erwähnt wird, ohne daß ibr Verhältnis zu den übrigen primären Qualitäten von Locke selbst recht klargestellt würde.

„Erste Qualität" ist also - trotz voller Abhängigkeit Lockes von Boyle in der Sache - terminologisch eine, freilich naheliegende, Neuerung Lockes. Aber auch hinsichtlich der "sekundären Qualitäten“ stimmen beide keineswegs völlig überein. Ursache davon ist eine unverkennbare Verschiebung des Sprachgebrauchs hinsichtlich des Wortes "Qualität". Bei Boyle haftete dem Ausdruck die unmittelbare Beziehung auf das Subjektive an, im Gegensatz zu den objektiven „Akzidenzien“, „Affektionen“, „Attributen“, „Modi“. Zwar unterscheidet auch er bei den sekundären Qualitäten zwischen der Vorstellung in uns (idea) und der besonderen Modifikation der Größe, Figur usw., die jener als realer Gehalt entspricht ${ }^{64}$ ); aber er trägt doch kein Bedenken, die Farben, Töne, Gerüche usw. in der Art, wie wir sie empfinden, als „Qualitäten ${ }^{4}$ zu bezeichnen ${ }^{65}$ ). Anderș Locke. Für jene subjektive Seite hat, er prinzipiell das Wort „Idee" vorbehalten (II 8,8 ). Im Unterschiede davon bezeichnet die "Qualität“ das Objektive, die Kraft, welche jene Vorstellungen in uns hervorruft $\left.{ }^{66}\right)$. Denn wenn auch unser Wissen im strengen Sinne auf unsere Vorstellungen und deren Verhältnisse beschränkt ist (IV 1), so haben wir doch auf Grund der Kausalität ${ }^{67}$ ) eine, obgleich weniger gewisse Auffassung (perception IV 2, 14) von den äußeren Dingen, die durch ihre Kräfte auf dem Wege des Stoßes (impulse II 8, 11 und 12) die Erregungen in unserem Gehirn und dadurch die Vorstellungen in unserem Geiste, die mit jenen nach der Einrichtung des Schöpfers (II 8, 13. IV $4,4)$ verbunden sind, hervorbringen. Sonach besteht wenigstens

6) S. oben Anm. 56 .

65) Vgl. Anm. 56 u. 57. Ferner Origin S. 466 a.

$\left.{ }^{66}\right)$ Die Ausnahme in dem Marginale zu II 23, 11 kann nicht in Betracht kommen.

$\left.{ }^{67}\right)$ Locke begründet das Kausalgesetz analytisch: Works III $60 \mathrm{f}$. 
eine Korrelation (dies der Sinn der „conformity“ IV 4, 4) zwischen unseren Vorstellungen einerseits und den Dingen und deren Zuständen andererseits. Den subjektiven „Ideen“ entsprechen die objektiven „Qualitäten“ (II 8, 8). Hierbei verhält nun eine gewisse Kंasse von Vorstellungsinhalten sich zu den ihnen zugrunde liegenden objektiven Eigentümlichkeiten (real qualities II 8, 17 u. ö.) wie Abbilder (images or representations II 30,2$)$ zu ihren Mustern (patterns II 8, 15). Die spezifischen Sinnesempfindungen dagegen sind den sie verursachenden Qualitäten so unähnlich, wie der Schmerz der ihn veranlassenden äußeren Ursache (II 8, 18).

Aber wie verhalten sich in den Dingen die sekundären zu den primären Qualitäten? Schon Boyle ließ die sekundären Qualitäten durch die Modifikationen der primären Affektionen entstehen. Allein bei ihm waren die „Qualitäten" die Empfindungsinhalte. Er wiederholte einfach die alte, schon bei Demokrit entwickelte Lehre. Locke, der die primären Affektionen primäre „Qualitäten“ nennt, unter den sekundären Qualitäten aber die realen Kräfte versteht, welche jene Empfindungen hervorrufen, hält Boyles Grundauffassung bei, gibt ihr aber, jenen Verschiebungen entsprechend, eine neue Wendung. Wenn er die sekundären Qualitäten (ebenso wie die tertiären, II 8,23 ) auf die primären Qualitäten zurückführt (IV $3,11)$, so bedeutet das bei ihm, daß die in den Dingen selbst real vorhandenen Kräfte durch die mannigfaltigen Verbindungen (combinations II 8, 22) oder Modifikationen (modifications II 8, 23) der primäı en Qualitäten konstituiert werden (vgl. auch II 8, 18), mag auch die Art dieser Konstitution und damit der Zusammenhang der sekundären Qualitäten untereinander und mit den primären uns verborgen bleiben (IV 3, 14; vgl. Works III S. 77).

Damit ist Locke merkwürdigerweise in gewisser Beziehung unvermerkt zu der aristotelisch-scholastischen Auffassung vom Verhältnisse der primären und sekundären Qualitäten zurückgekehrt, wie wir diese in durchgebildeter Gestalt bei Heinrich von Hessen kennen gelernt haben. Freilich mit einem charakteristischen Unterschied. Wenn wir in der üblichen Weise - nicht der Lockeschen Terminologie gemäß - den Gegensatz der von Galilei, Hobbes und Descartes durchgeführten mechanischen Physik gegenüber der aristo- 
telisch-scholastischen Naturphilosophie dahin bestimmen, daß jene an die Stelle der qualitativen Naturbetrachtung der Scholastik eine quantitative Erklärungsweise setzt („mechanisch" sagte Boyle), so finden wir die Folgen dieser durchgreifenden Veränderung der wissenschaftlichen Grundlegung auch in dem gänzlich verschiedenen Inhalt, den trotz aller Ähnlichkeit der formalen Betrachtung die primären und sekundären Qualitäten bei Locke und in der Scholastik haben. Als räumliche Bestimmungen und mechanisch wirkende Kräfte sind Lockes erste (qualitäten und die in den Kombinationen dieser ersten Qualitäten bestehenden zweiten Qualitäten nicht mehr qualitativ im ursprünglichen Sinne, sondern tragen den Charakter des Quantitativen. So spiegelt sicli auch in dieser Einzelheit der allgemeine Umschwung, den die Entwickelung der neuen, mathematisch-mechanisch orientierten Wissenschaft herbeiführte.

Stellen wir das Ergebnis kurz zusammen, so stellt sich uns die Geschichte der Terminologie der ersten und zweiten Qualitäten folgendermaßen dar.

Nicht von ersten Qualitäten, wohl aber von ersten Differenzen unter den Qualitäten spricht schon Aristoteles. Er versteht darunter die zwei Gegensatzpaare unter den Tastqualitäten: Warm Kalt, Trocken Feucht, die durch ihre verschiedenen Kombinationen die ursprünglichen Unterschiede der Körper, nämlich den Unterschied der vier Elemente, begründen. „Erste Qualitäten“ (qualitates primae oder primariae) werden diese Gegensätze in der arabischen und lateinischen Philosophie des Mittelalters genannt, in der letzteren schon allgemein im XIII. Jahrhundert. Die übrigen Tastqualitäten sowie die Qualitäten der vier anderen Sinne (die des Gehörssinns mit Einschränkungen) werden in Übereinstimmung mit Aristoteles oder doch in Weiterführung seiner Gedanken auf diese ersten Qualitäten zurückgeführt, nicht als deren subjektive Wirkungen in uns, sondern als abgeleitete objektive Beschaffenheiten. So unterscheidet schon Albert der Große prima sensibilia und secunda sensibilia. Dafür werden dann - nicht erst bei Bartholomaeus von Usingen, sondern nachweisbar schon im XIV. Jahrhundert bei 
Heinrich von Hessen - auch die Ausdrücke „qualitates primae" und "qualitates secundae" üblich. In dieser Form ist die scholastische Lehre noch im Jahrhundert Lockes allverbreitet. Auch aus Gassends Polemik ist sie bekaunt.

Die neue mathematisch-mechanische Naturphilosophie des XVII. Jahrhundert führt zu einer Erneuerung der schon in der antiken Atomistik vorgebildeten und im Anschluß an diese von Gassend aufgenommenen Anschauung, die Aristoteles dahin charakterisiert hatte, daß sie die spezifischen Sinnesqualitäten auf die gemeinsamen Wahrnehmungsinhalte: Größe, Figur, Zahl, Bewegung und Ruhe zurückführe (zu denen noch die Undurchdringlichkeit als Natur der raumfüllenden Materie hinzutrat). Als „erste Akzidenzien“ stellt Galilei diese den Qualitäten gegenüber, welche erst durch die Einwirkung jener auf die Sinne entstehen und darum nach ihm, wie nach Descartes, Hobbes, Gassend, subjektiver Natur sind. Für diese subjektiven sinnlichen Qualitäten verwendet dann Robert Boyle den scholastischen Terminus „sekundäre Qualitäten“; die realen Eigenschaften dagegen nennt er zwar, wie Galilei, „erste Akzidenzien “, auch „erste Affektionen“ u. dgl., aber noch nicht „erste Qualitäten“. Locke führt die scholastische Bezeichnung "erste und zweite Qualitäten" ein, indem er zugleich die ersten Qualitäten mit den "gemeinschaftlichen Wahrnehmungsinhalten “ des Aristoteles (mit der angegebenen Erweiterung) gleichsetzt. Aber die "Qualität" hat bei ihm nicht mehr denselben Sinn wie bei Boyle. Indem er eine bei Descartes, Boyle u. a. sich findende Unterscheidung strenger durchführt, stellt er die "Idee* - welche bei Boyle die von den realen Akzidenzien verschiedene Qualität selbst war - als das Subjektive der "Qualität" als dem Objektiven gegenüber. Darum sind "sekundäre Qualitäten" bei Locke nicht, wie bei Boyle, die „Ideen", d. h. die Sinnesinhalte selbst; vielmehr versteht er darunter die realen Kräfte in den Dingen, welche jene Ideen bewirken, ihnen aber unähnlich sind. Deshalb kann er - wieder nach einer Anregung bei Boyle - den sekundären Qualitäten noch „tertiäre" zur Seite stellen (Essay II 8, 23): die „gewöhnlich so genannten Kräfte", welche Ursachen der Veränderungen nicht in unseren Organen, sondern in anderen von 
uns wahrgenommenen Körpern sind. Die sekundären Qualitäten aber werden (wie die tertiären) durch die mannigfachen Verbindungen der primären konstituiert, nicht im Bewußtsein, sondern in den Dingen selbst. So gleichen in formaler Beziehung die primären und sekundären Qualitäten Lockes mehr denen der Scholastik, als denen Boyles; ibr Inhalt aber ist, entsprechend der neuen Wissenschaft, ein anderer geworden.

Aus dem Gesagten erhellt, daß die landläufige Darstellung in mehreren Punkten zu korrigieren ist. Vor allem in zwei Stücken. Das erste Vorkommen der scholastischen T'ermini „qualitates primae ${ }^{“}$ und „qualitates secundae“ setzt sie um zwei Jahrhunderte zu spät an. Boyle gegenüber aber ist, trotz aller sachlichen Abhängigkeit, in terminologischer Beziehung Locke doch selbständiger, als sie annimmt. Die „primären Qualitäten “ hat Locke überhaupt nicht von Boyle, die „sekundären“ aber haben bei ihm einen anderen Sinn als bei jenem ${ }^{68}$ ).

\section{Nachtrag zum ersten Artikel.}

Wenn auch Locke, wie im ersten Artikel bemerkt wurde, im Essay nicht von einer "tabula rasa" spricht, sondern von einem "white paper, void of all characters", so hat die übliche Darstellung doch ganz recht, wenn sie Lockes Meinung mit dem so bezeichnenden scholastischen Ausdruck charakterisiert. Sie folgt darin nur Lockes eigenem Beispiel. Der Auszug aus seinem Essay, welchen er im Jahre 1687 für Le Clerc verfaßte und den dieser in französischer Übersetzung in der „Bibliothèque universelle et historique", tome VIII", Amsterdam 1688, veröffentlichte, beginnt

68) Eine Analyse der Lockeschen Theorie nach ihren inneren Motiven unter historischem Gesichtspunkte habe ich im Philosophischen Jahrbuch, Bd. XXI, Fulda 1908, S. 293-313 gegeben („Über die Lockesche Lehre von den primären und sekundären Qualitäten"). Dort zeige ich auch S. $311 \mathrm{ff}$. nach Robert Boyles Abbandlung „On the Systematical or Cosmical Qualities“ (1669), wie die von Fraser und anderen völlig uniBverstandene rätselhafte Äußerung Lockes (Essay IV 3, 11) über eine Ursache der sekundären Qualitäten, die unserer Erfahrung noch ferner steht, als die primären Qualitäten, $\mathrm{zu}$ verstehen ist. 
dort S. 49 mit den für Lockes Philosophie seitdem gewöhnlich als programmatisch betrachteten Worten: „Dans les pensées que j'ai euës, concernant nôtre Entendement, j'ai tâché d'abord de prouver que nôtre Esprit est au commencement ce qu'on appelle tabula rasa; c'est à dire, sans idées et sans connoissance". Den Wortlaut des englischen Originals hat Lord King, The Life of John Locke, London 1830, Bd. II, S. 231, mit geteilt. Dort heißt es: „In the thoughts I have had concerning the Understanding, I have endeavoured to prove that the mind is at first rasa tabula." Die im Französischen hinzugesetzte Erklärung der „tabula rasa“ hat Locke also, wenigstens ursprünglich, nicht für nötig gebalten.

Auch sonst ist das Bild der "tabula rasa" der englischen Philosophie nicht fremd. So schreibt Hobbes in Leviathan c. 47 (ed. Molesworth, Latin III p. 508 - im englischen Text fehlt die Stelle): Si cordibus scripsissem instar tabularum rasarum puris, brevior esse potuissem.

Noch sei hervorgehoben, daß die S. $297 \mathrm{f}$. gebotenen Zitate aus älteren Scholastikern, wie dort schon bemerkt wurde, nur die nötigsten Beispiele als Beleg geben sollen. Sonst hätte ich noch manche andere Stellen anführen können: Albertus Magnus, Summa de creaturis I q. 24 a. 2 , Bd. XXXIV S. 478 b, Bonaventura II Sent. d. 3 p. 2 a. 2 q. 1 f. 5 , pag. 118 a usw. 